ISSN 0103-9954

\title{
EFEITO DO FOGO SOBRE A RIQUEZA DE FORMIGAS (HYMENOPTERA: FORMICIDAE) ASSOCIADAS À Pinus elliottii ENGELM. NO SUL DO BRASIL
}

\author{
THE EFFECT OF FIRE OVER RICHNESS OF ANTS (HYMENOPTERA: FORMICIDAE) \\ ASSOCIATED TO Pinus elliottii ENGELM. IN SOUTHERN BRAZIL
}

\author{
Jardel Boscardin $^{1}$ Ervandil Corrêa Costa ${ }^{2}$ Jacques Hubert Charles Delabie ${ }^{3}$ Juliana Garlet $^{4}$
}

\begin{abstract}
RESUMO
Este trabalho objetivou avaliar o efeito do fogo sobre a fauna de formigas associada à Pinus elliottii Engelm. $\mathrm{O}$ estudo foi desenvolvido no município de Santa Maria, Rio Grande do Sul, numa área experimental junto ao Campus da Universidade Federal de Santa Maria (UFSM), em um plantio de um hectare de Pinus elliottii com 28 anos de idade e espaçamento de $2 \mathrm{~m}$ x $2 \mathrm{~m}$, no qual ocorreu um incêndio ao final de novembro de 2008. Um mês após, iniciou-se uma amostragem da fauna de formigas, concluída em novembro de 2009. Utilizou-se o método de interceptação de insetos perambulantes através do uso de armadilhas de solo sem atrativo, distribuídas no centro do povoamento, sendo dispostas 10 armadilhas em transecto linear simples, em intervalos de 10 metros permanecendo enterradas por 48 horas. Ao final deste período, o material biológico foi acondicionado em potes plásticos e encaminhado ao laboratório para triagem, onde as formigas foram separadas em morfoespécies e prosseguiram para identificação. A partir dos levantamentos foram encontradas 25 espécies de formigas distribuídas em onze gêneros, oito tribos e quatro subfamílias. Destas, as que apresentaram maiores frequências de ocorrência em suas respectivas subfamílias foram Pseudomyrmex termitarius (46,7\%), Acromyrmex crassispinus e Pachycondyla striata (ambas com 35,8\%), e Camponotus blandus (25,0\%). O fogo é um agente de distúrbio ambiental por vezes significativo, causando efeitos positivos e negativos sobre a fauna edáfica. Sua ação pode ter gerado efeito indireto negativo sobre Crematogaster victima e positivo sobre Pseudomyrmex termitarius, Acromyrmex crassispinus, Pachycondyla striata e Camponotus blandus presentes no sub-bosque de Pinus elliottii, pós-incêndio.
\end{abstract}

Palavras-chave: entomologia florestal; incêndio florestal; insetos bioindicadores.

\section{ABSTRACT}

This work aimed at assessing the effect of fire over an ant fauna associated to Pinus elliottii Engelm. The study was developed in the city of Santa Maria, Rio Grande do Sul, in an experimental area next to the Campus of Universidade Federal de Santa Maria (UFSM), in a 28-year-old plantation of an hectare of Pinus elliottii, spacing $2 \mathrm{~m} \times 2 \mathrm{~m}$, on which a fire happened in the end of November, 2008. One month later, an ant fauna sampling was initiated, being concluded in November, 2009. The method of interception of wandering insects has been used through soil traps without attraction, distributed in the center of the population, being prepared 10 traps in simple linear transection, in intervals of 10 meters that remained buried for 48 hours. After such period, the biological material was packed in plastic pots, transported to the laboratory for a triage, where the ants were separated into morphospecies and proceeded to identification.

1 Engenheiro Florestal, Msc., Doutorando do Programa de Pós-Graduação em Engenharia Florestal da Universidade Federal de Santa Maria, Av. Roraima, 1000, CEP 97105-900, Santa Maria (RS), Brasil. boscardinj@gmail.com

2 Engenheiro Agrônomo, Dr., Professor do Programa de Pós-Graduação em Engenharia Florestal da Universidade Federal de Santa Maria, Av. Roraima, 1000, CEP 97105-900, Santa Maria (RS), Brasil. ervandilc@gmail.com

3 Biólogo, Dr., Pesquisador da Comissão Executiva do Plano da Lavoura Cacaueira do Cacau, Av. Juca Leão, 463, CEP 45600-770, Itabuna (BA), Brasil. jacques.delabie@gmail.com

4 Engenheira Florestal, Dr ${ }^{\text {a }}$, Professora Adjunta da Faculdade de Ciências Biológicas e Agrárias da Universidade do Estado de Mato Grosso, Campus de Alta Floresta, Av. Perimetral Rogério Silva, s/n, CEP 78580-000, Alta Floresta (MT), Brasil. julianagarlet@unemat.br

Recebido para publicação em 9/06/2012 e aceito em 24/04/2013 
From the surveys, 25 ant species were found, distributed in eleven genera, eight tribes and four subfamilies. From these, the ones who presented the biggest occurrence frequencies in their respective subfamilies were Pseudomyrmex termitarius (46,7\%), Acromyrmex crassispinus, and Pachycondyla striata (both with 35,8\%), and Camponotus blandus (25,0\%). Fire is an agent of environmental disturbance many times significant, causing positive and negative effects over the edaphic fauna. Its action might have caused a negative indirect effect over Crematogaster victima and positive over Pseudomyrmex termitarius, Acromyrmex crassispinus, Pachycondyla striata and Camponotus blandus present in the understory of Pinus elliottii, after the fire.

Keywords: forest entomology; forest fire; bioindicator insects.

\section{INTRODUÇÃO}

Pinus elliottii Engelm. é uma gimnosperma da família Pinaceae originária do sudeste dos Estados Unidos, onde é tida como sensível ao fogo; apresenta excelente crescimento nas regiões de clima subtropical úmido, sendo muito cultivada no sul do Brasil, possui fibras longas adequadas para a fabricação de papel (MARCHIORI, 1996). Sua madeira destina-se também ao processamento mecânico e à extração de resina (SHIMIZU, 2006).

Em áreas cultivadas com pináceas observase um grande acúmulo de serapilheira, formado pelas acículas caídas das árvores. Isto porque, segundo Kleinpaul et al. (2005), as acículas possuem alto teor de lignina, o que dificulta a atividade de organismos decompositores. Desta forma, o acúmulo de serapilheira sobre a superfície do solo the confere elevado risco de fogo (LORO e HIRAMATSU, 2004).

De acordo com Pippi et al. (2000), incêndios florestais ocorrem em todo o mundo, onde extensas áreas de florestas são atingidas pelo fogo devido à irresponsabilidade humana, e por vezes, pela ação da própria natureza, podendo causar impactos irreparáveis aos ecossistemas e prejuízos para as populações de espécies da fauna e da flora locais. Nesse sentido, para punir quem provoca incêndio criminoso em mata ou floresta, a legislação brasileira prevê no Art. 41 da Lei n. 9.605, de 12 de fevereiro de 1998, pena de reclusão de dois a quatro anos, e multa (BRASIL, 1998).

O fogo é um distúrbio que pode afetar a riqueza das comunidades bióticas através de efeitos diretos ou indiretos. Os efeitos diretos são: mortes, queimaduras e intoxicações. Já os indiretos tendem a ser mais amplos, tardios e diversos, sendo as alterações ocasionadas na estrutura das comunidades animais, provenientes de modificações que ocorrem na paisagem (FRIZZO et al., 2011).

Desta forma, segundo Araújo e Ribeiro (2005), a ação do fogo causa impactos à fauna do solo, e tais alterações irão depender do tipo e da intensidade do fogo, bem como das variáveis ambientais e da adaptação dos organismos. Isso pode resultar na redução ou no aumento da abundância e sucessão ecológica desses animais.

A fauna edáfica presente na serapilheira responde às perturbações do ambiente, pelo fato de apresentar uma codependência dos processos que ocorrem no sistema serapilheira-solo (CORREIA e PINHEIRO, 1999). Nesse sentido, o fogo pode causar um impacto imediato, através da mortalidade direta; em longo prazo, os artrópodes que habitam o solo respondem aos seus efeitos em função da alteração dos processos ecológicos fundamentais, tais como a ciclagem de nutrientes e a produção primária (MILLER et al., 1955).

Devido a esta sensibilidade, as modificações do meio onde vive a fauna edáfica, ou determinados grupos que dela fazem partes, vêm sendo utilizados como biodindicadores das alterações causadas pelo fogo em diversos ecossistemas (OLIVEIRA e FRANKLIN, 1993; NUNES et al., 2009; VASCONCELOS et al., 2009).

As formigas são tidas como potenciais indicadores da qualidade ambiental, por apresentarem muitas características esperadas dos bioindicadores, como: abundância local alta, alta riqueza local e global, possuírem muitos táxons especializados, facilidade nas amostragens e separação em morfoespécies, assim como por serem organismos sensíveis às mudanças do ambiente (MAJER, 1983). Desse modo, estudos da biodiversidade e dinâmica, desenvolvidos com esse grupo em ambientes alterados permitem o entendimento das perturbações ambientais ocorridas (MAJER, 1996; RIBAS et al., 2012). Por este motivo, o grupo das formigas tem sido utilizado, em vários estudos como indicador dos efeitos decorrentes do fogo (COELHO et al., 2007; FRIZZO et al., 2007; LOPES et al., 2007; SANTOS et al., 2008).

Os incêndios florestais podem ser facilmente 
mensuráveis economicamente, quando atingem a produção florestal ou agrícola e, imensuráveis, nesse sentido, quando se tratam dos impactos causados pelo fogo sobre a biodiversidade animal e vegetal associada a tais culturas. Restando para esses últimos, estudos que indiquem os efeitos sobre o comportamento das espécies. Assim, o presente estudo objetivou avaliar o efeito do fogo sobre a fauna de formigas associada a um plantio de Pinus elliottii na região central do Rio Grande do Sul, Brasil.

\section{MATERIAIS E MÉTODOS}

\section{Caracterização e localização da área de estudo}

O estudo foi conduzido no município de Santa Maria, localizado na região fisiográfica da Depressão Central do Estado do Rio Grande do Sul. Possui clima temperado chuvoso e quente que se enquadra na categoria "Cfa" na classificação de Köppen. As precipitações são regulares ao longo do ano, com índices pluviométricos anuais de 1500 a $1750 \mathrm{~mm}$ (PEREIRA et al., 1989). Apresenta temperatura média do mês mais quente superior a $22^{\circ} \mathrm{C}$, e a temperatura do mês mais frio entre $-3^{\circ} \mathrm{C}$ e $18^{\circ} \mathrm{C}$ (MORENO, 1961).

$\mathrm{O}$ experimento foi instalado em uma área experimental de um hectare, plantada com Pinus elliottii, em espaçamento de $2 \mathrm{~m} \times 2 \mathrm{~m}$, implantado no ano de 1981 (PES et al., 1995). Nessa área $\left(29^{\circ} 42^{\prime} \mathrm{S} 53^{\circ} 42^{\prime} \mathrm{O}\right.$, altitude aproximadamente $\left.95 \mathrm{~m}\right)$ ocorreu um incêndio, sem causa conhecida, ao final de novembro de 2008. A área encontra-se inserida ao Campus da Universidade Federal de Santa Maria (UFSM), e pertence ao Departamento de Ciências Florestais.

O fogo durou por um dia, consumindo a serapilheira existente na área. As chamas não atingiram os galhos nem a copa das árvores. Verificaram-se marcas de carbonização nas cascas das árvores de Pinus elliottii, até um metro de altura a partir do nível do solo. Troncos e galhos mais grossos (com diâmetros superiores a $10 \mathrm{~cm}$ ) de árvores, que se encontravam caídos sob o plantio, também foram acometidos pela ação do fogo, porém, verificou-se carbonização somente da casca.

\section{Amostragem da mirmecofauna}

Para amostragem da fauna de formigas, foi escolhido o método por interceptação de insetos perambulantes (fauna epigeica), utilizando-se armadilhas de solo, adotadas, pela simplicidade, baixo custo, e por possuir ação uniforme no ambiente, amostrando tanto espécies diurnas quanto noturnas (MAJER, 1997). Assim, instalaram-se armadilhas de tipo pitfall, adaptadas do modelo sugerido por Almeida et al. (2003), compostas por um recipiente plástico de $10 \mathrm{~cm}$ de altura, área de captura de $78,5 \mathrm{~cm}^{2}$ e capacidade para $500 \mathrm{~mL}$. Como conservante, utilizou-se uma solução heterogênea formada por $200 \mathrm{~mL}$ de água, cinco gotas de detergente líquido e 10 gramas de cloreto de sódio. As armadilhas foram enterradas no solo de maneira que ficassem com a borda ao nível da serapilheira. Como proteção contra a chuva, e queda de acículas e sementes, utilizou-se um prato plástico, do tipo descartável, de $176,7 \mathrm{~cm}^{2}$ fixado ao solo com três ganchos de arame, a aproximadamente $10 \mathrm{~cm}$ acima da serapilheira.

As armadilhas foram posicionadas no interior do povoamento, de maneira a minimizar um possível efeito de borda, sendo dispostas 10 armadilhas em transecto linear simples, a intervalos de 10 metros. Essa metodologia é sugerida por Sarmiento-M (2003) com a finalidade de padronizar os levantamentos realizados com a família Formicidae, a fim de que se possam comparar diferentes estudos, uma vez que, segundo o mesmo autor, não existiriam variações na composição de formigas em distâncias de até 100 metros, dentro de uma área de floresta.

As séries de coletas foram realizadas mensalmente, no período de 16 de dezembro de 2008 a 20 de novembro de 2009, totalizando 12 ao final do período de um ano de amostragem. As armadilhas permaneceram expostas por um período de 48 horas (SARMIENTO-M, 2003), sendo que, ao final deste período, o material coletado foi acondicionado em potes plásticos, devidamente rotulados, e em seguida levado ao Laboratório de Entomologia Florestal do Centro de Ciências Rurais da UFSM. Em laboratório procedeu-se à triagem do material, separando as formigas em nível de gênero, a partir da chave dicotômica de Bolton (1994) e em morfoespécies. Em seguida, espécimes foram enviados ao Laboratório de Mirmecologia do Centro de Pesquisas do Cacau, Ilhéus, Bahia, para identificação.

\section{Análise dos parâmetros ecológicos}

A partir dos dados levantados, realizou- 
TABELA 1: Riqueza de espécies $(S)$ e frequência de ocorrência, absoluta $(\mathrm{N})$ e relativa $(\%)$, de formigas capturadas em armadilhas de solo associadas à Pinus elliottii pós-incêndio em Santa Maria RS, Brasil (2008/2009).

TABLE 1: Species richness $(S)$ and absolute $(\mathrm{N})$ and relative (\%) frequency of occurrence of ants captured by pitfall traps associated with Pinus elliottii after fire in Santa Maria, RS state, Brazil (2008/2009).

\begin{tabular}{lc}
\hline \multicolumn{1}{c}{ Subfamília/ Tribo/ Espécie } & $\mathrm{N}(\%)^{1}$ \\
\hline \multicolumn{1}{c}{ FORMICINAE $(S=7)$} & \\
\hline Camponotini & $30(25,0)$ \\
Camponotus blandus (Fr. Smith, 1858) & $2(1,7)$ \\
Camponotus (Myrmaphaenus) sp.1 & $12(10,0)$ \\
Camponotus melanoticus Emery, 1894 & $21(17,5)$ \\
Camponotus mus Roger, 1863 & $2(1,7)$ \\
Camponotus novogranadensis Mayr, 1870 & $1(0,8)$ \\
Camponotus renggeri Emery, 1894 & $8(6,7)$ \\
Camponotus rufipes (Fabricius, 1775) & \\
\hline
\end{tabular}

MYRMICINAE $(S=14)$

\section{Attini}

Acromyrmex crassispinus Forel, 1909

$43(35,8)$

Cyphomyrmex daguerrei Santschi, 1933

$1(0,8)$

Mycocepurus goeldii Forel, 1893

$7(5,8)$

Crematogastrini

Crematogaster victima Forel, 1901

Myrmicini

Pogonomyrmex naegelii Forel, 1878

Pheidolini

Pheidole alpinensis Forel, 1912

$3(2,5)$

Pheidole gigas Wilson, 2003

$4(3,3)$

Pheidole jelskii Mayr, 1884

Pheidole perryorum Wilson, 2003

$12(10,0)$

Pheidole radoszkowskii Mayr, 1884

$17(14,2)$

Pheidole sp. prox. Sigillata

$4(3,3)$

Pheidole sp.2 gp. Pallidens

$22(18,3)$

Solenopsidini

Solenopsis invicta Buren, 1972

$4(3,3)$

Solenopsis sp.

$6(5,0)$

PONERINAE $(S=3)$

\section{Ponerini}

Odontomachus chelifer (Latreille, 1802)

Pachycondyla harpax (Fabricius, 1804)

Pachycondyla striata (Smith, 1858)

$43(35,8)$

\section{PSEUDOMYRMECINAE $(S=1)$}

Pseudomyrmicini

Pseudomyrmex termitarius (Smith, 1855)

$56(46,7)$

Em que: ${ }^{1}$ Número de armadilhas de solo em que a espécie foi encontrada. 
se uma análise dos parâmetros ecológicos de frequência de ocorrência e riqueza de espécies de formigas, bem como uma avaliação da flutuação populacional das espécies mais representativas.

Para evitar a superestimativa, devido ao efeito do tamanho das colônias, os dados de abundância foram calculados com base na frequência de ocorrência das espécies nas armadilhas e não quanto ao número de indivíduos coletados (ROMERO e JAFFÉ, 1989; LONGINO, 2000). Nesse sentido, a frequência de ocorrência absoluta constituiu-se do número de armadilhas em que foi encontrada determinada espécie, enquanto que a frequência de ocorrência relativa é calculada com base na frequência absoluta, utilizando-se a equação $\%=\mathrm{N} / \mathrm{n} * 100$, em que $\%=$ frequência de ocorrência relativa, $\mathrm{N}$ = frequência de ocorrência absoluta, e $\mathrm{n}=$ corresponde ao número de armadilhas de solo utilizadas ao longo do estudo, neste caso 120.

A riqueza $(S)$ consiste em uma medida ou contagem do número de espécies encontrado em determinada área (RICKLEFS, 1996). Para estimar a riqueza de espécies presentes na área em estudo foi utilizado o índice de Jackknife $1^{\text {a }}$ Ordem, que, segundo Palmer (1990), é um dos índices não paramétricos mais precisos para estimar a riqueza de uma comunidade. Este foi calculado com o auxílio do programa EstimateS versão 7.5.2 (COLWELL, 2009), levando-se em consideração cada data de coleta como uma amostra, totalizando, assim, 12 amostras. Ainda, com base nas frequências de ocorrência nas armadilhas de solo observou-se a flutuação populacional das espécies mais abundantes em cada subfamília, expressa na forma gráfica.

\section{RESULTADOS E DISCUSSÃO}

A partir dos levantamentos foram identificadas 25 espécies de formigas distribuídas em onze gêneros, oito tribos e quatro subfamílias (Tabela 1).

O índice de Jackknife de $1^{\text {a }}$ Ordem estimou em 29,58 o número de espécies que seriam capturadas nas armadilhas de solo se a amostragem fosse continuada infinitamente; portanto, obteve-se, com base nesta metodologia de amostragem, cerca $84 \%$ da comunidade total estimada (Figura 1).

Em uma área da floresta amazônica, após 15 anos de incidência de fogo, Santos et al. (2008) verificaram uma configuração similar quanto ao número de subfamílias encontradas

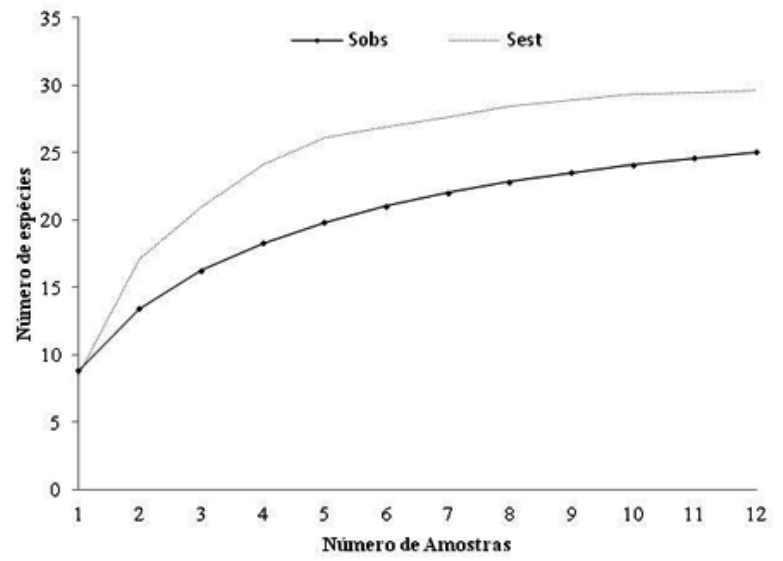

FIGURA 1: Curva cumulativa de riqueza de espécies de formigas observadas $\left(S_{\text {obs }}\right)$ e estimadas $\left(S_{\text {est }}\right)$ capturadas em armadilhas de solo associadas à Pinus elliottii após incêndio em Santa Maria - RS, Brasil (2008/2009). Método não paramétrico Jackknife de $1^{\text {a }}$ Ordem empregando-se 100 aleatorizações (EstimateS v. 7.5.2).

FIGURE 1: Cumulative curve of observed $\left(S_{\text {obs }}\right)$ and estimated $\left(S_{\text {est }}\right)$ ant species richness in pitfall traps associated with Pinus elliottii after fire in Santa Maria, RS state, Brazil (2008/2009). Non-parametric method Jackknife first order employing 100 randomizations (EstimateS v.7.5.2).

(seis): Myrmicinae $(S=56)$, Formicinae $(S=1)$, Dolichoderinae $(S=9)$, Ectatomminae $(S=5)$, Ponerinae $(S=4)$, e Paraponerinae $(S=1)$. No presente estudo, não ocorreram Ectatomminae, Paraponerinae e Dolichoderinae, e sim Pseudomyrmecinae.

Nos mais diversos ecossistemas, assim como ocorreu no presente estudo, Myrmicinae constitui-se como a maior e mais diversa subfamília de Formicidae, tanto na escala regional ou global (HÖLLDOBLER e WILSON, 1990). Crematogaster victima (Myrmicinae) foi encontrada nas amostragens em uma única amostra da última serie no mês de novembro de 2009, ou seja, um ano após a ocorrência do incêndio. Este fato pode estar ligado ao efeito negativo indireto do fogo, através da destruição de abrigo, da alimentação, e da consequente exposição de Crematogaster victima à ação de predadores. Porém, sugerem-se estudos nesse sentido, em trabalhos futuros. 


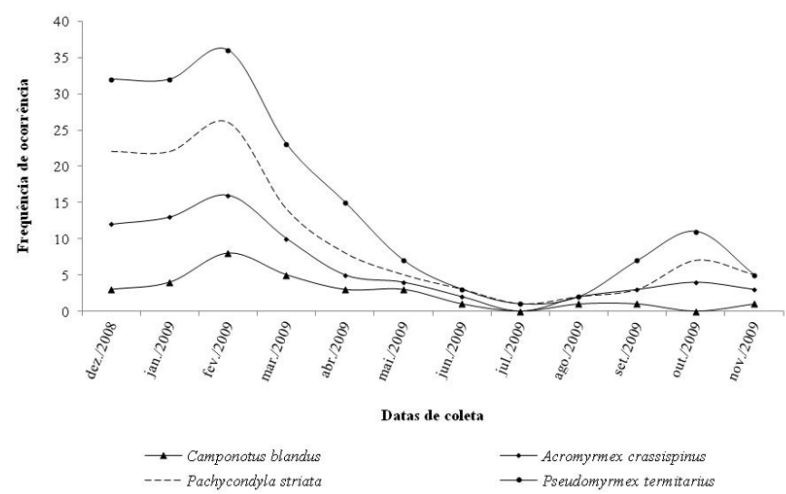

FIGURA 2: Flutuação populacional de quatro espécies, representantes das subfamílias de Formicidae (Hymenoptera) associadas a um povoamento, pósincêndio, de Pinus elliottii, em Santa Maria - RS, Brasil (2008/2009).

FIGURE 2: Fluctuation of four species representatives of the subfamilies of Formicidae (Hymenoptera) associated with a settlement, after fire, of Pinus elliottii in Santa Maria, RS state, Brazil (2008/2009).

Em contrapartida, Acromyrmex crassispinus, apresentou-se como a mais abundante entre as Myrmicinae, com ocorrência de 35,8\% (Tabela 1). Esta espécie, conhecida popularmente como "formiga-de-cisco" é comumente encontrada na Região Sul do Brasil (RANDO e FORTI, 2005). Alguns estudos mostram que espécies de Acromyrmex, incluindo Acromyrmex crassispinus, causam danos significativos em plantas jovens de espécies pertencentes ao gênero Pinus com dois a três meses de idade (LINK et al., 2001). Este fato pode explicar a flutuação populacional verificada para Acromyrmex crassispinus (Figura 2), uma vez que, dois meses após a ocorrência do incêndio, observou-se intensa brotação de sementes de Pinus elliottii no sub-bosque do povoamento, provocada possivelmente, pela quebra de dormência das sementes pela ação do fogo e remoção da camada de serapilheira que permitiu a fixação da radícula da semente germinada ao solo mineral, o que resultou, em um "tapete" de plântulas (Figura 3), aumentando assim, a disponibilidade de recursos para esta espécie que cultiva um fungo simbiótico com esse substrato. Nesse sentido, o fogo apresentou um efeito positivo indireto sobre Acromyrmex crassispinus.

$\mathrm{Na}$ subfamília Formicinae predominaram espécies do gênero Camponotus, tendo sido

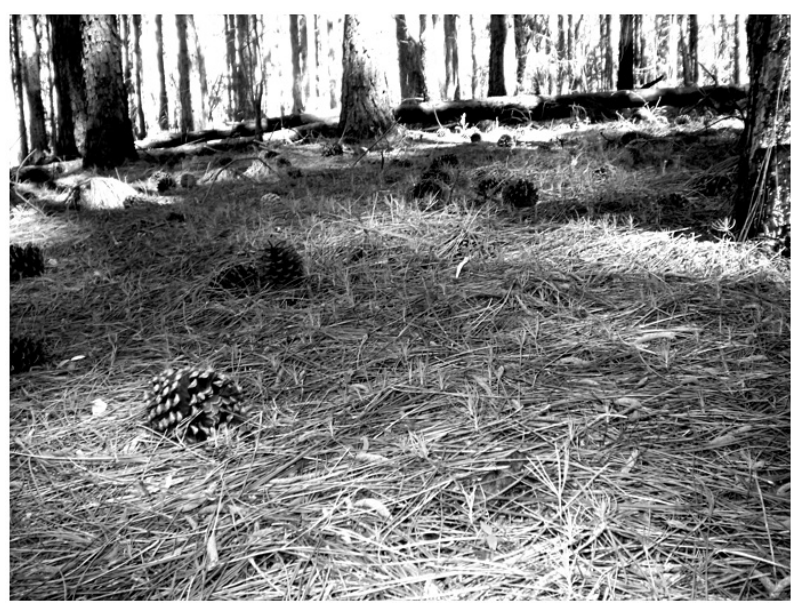

FIGURA 3: "Tapete" de plântulas no sub-bosque de Pinus elliottii, em janeiro de 2009, dois meses após incêndio, Santa Maria - RS, Brasil.

FIGURE 3: 'Carpet' of seedlings in the understory of Pinus elliottii, in January 2009, two months after fire, Santa Maria, RS state, Brazil.

encontradas sete no total (Tabela 1). De acordo com Wilson (1976), espécies deste gênero, juntamente com as de Crematogaster e Pheidole, apresentamse como as mais abundantes e frequentes do mundo. Por sua vez, Camponotus blandus apresentou maior frequência de ocorrência dentro da subfamília Formicinae, sendo encontrada em 25,0 \% das amostras. Esta espécie é onívora e generalista, constitui-se como insaciável predadora de cupim e, possui ação predatória agressiva, invadindo as galerias de enxameagem dos termiteiros, apresentando forte redução populacional quando os cupins encontram-se numa fase crítica do ciclo de vida (SANTOS e RESENDE, 1996). Este fato pode explicar o aumento de Camponotus blandus após o incêndio, uma vez que, alguns troncos de árvores encontravam-se caídos e com a ação do fogo, as possíveis colônias de cupins teriam ficado expostas. Ressalta-se, no entanto, que não se levantou a presença desses insetos na área.

A subfamília Ponerinae é considerada o grupo de formigas mais primitivo, tanto por aspectos morfológicos como comportamentais. Conforme se observa na Tabela 1, Ponerinae apresentou neste estudo três espécies: Odontomachus chelifer, Pachycondyla harpax e Pachycondyla striata. A última citada é a mais abundante $(35,8 \%)$. Segundo 
Lattke (2003), espécies do gênero Pachycondyla (Tribo Ponerini) possuem distribuição pantropical e peritropical, sendo comumente encontradas em florestas úmidas onde predam sobre ou dentro do solo, ou na vegetação, porém, também habitam florestas secas e de galeria em savanas. Todas as espécies do gênero são predadoras, sendo algumas especialistas e outras generalistas, incluindo espécies mirmecocóricas (PETERNELLI et al., 2004).

Conforme pode ser observado na Figura 2, Pachycondyla striata manteve a segunda maior frequência de ocorrência, após Pseudomyrmex termitarius. Essa intensa atividade verificada por Pachycondyla striata, nos meses subsequentes aos da ocorrência do fogo, pode ser entendida, pelo comportamento alimentar detritívoro desta espécie, tendo em vista a quantidade de alimento ofertada após a ação do fogo, através de carcaças, e a falta de abrigo para os demais organismos favorecendo sua atividade de predação, e também porque no verão as populações estão mais resistentes e há mais operárias disponíveis para forragear. Segundo Gianotti e Machado (1994), Pachycondyla striata possui hábito generalista, coletando especialmente carcaças de insetos e predando cupins, formas jovens de artrópodes e minhocas. Medeiros (1997) verificou 132 itens na constituição da dieta das colônias de Pachycondyla striata, destes, 32,8\% constituíram-se de formigas adultas e suas formas imaturas (ovos, larvas e pupas), sendo que das formigas adultas consumidas, apenas 7,0\% estavam vivas.

Já Pseudomyrmecinae apesar de representada somente pela espécie Pseudomyrmex termitarius, esta, dentre todas as espécies coletadas, apresentou a maior frequência de ocorrência, com 46,7 \% (Tabela 1). Espécies desse grupo, geralmente têm comportamento onívoro e não patrulham em grandes grupos (SILVESTRE e SILVA, 2001). Segundo Silveste (2000), Pseudomyrmex termitarius nidifica em troncos caídos ou em cupinzeiros, o que pode explicar a alta frequência de ocorrência encontrada para esta espécie ao longo do ano de avaliação, já que seus locais de nidificação encontram-se protegidos da ação do fogo.

A partir da análise do gráfico de flutuação (Figura 2), percebe-se que as quatro espécies apresentaram uma maior frequência após a ocorrência do incêndio. Este fato parece estar relacionado aos efeitos indiretos do fogo, tanto positivos quanto negativos, sobre os hábitos alimentares e comportamentais das espécies de formigas.

O efeito do fogo sobre as espécies formigas depende da intensidade e comportamento do incêndio. Segundo York (1994), a riqueza de espécies de formigas tende a aumentar após a incidência do fogo, no entanto, também tende a diminuir com a perda desses efeitos sobre a fauna de formigas. No Cerrado brasileiro, uma diminuição da riqueza de formigas foi observada, devido, possivelmente, à eliminação de colônias e à escassez de alimento pela diminuição de recursos alimentares disponíveis nas plantas (LOPES et al., 2007). Outras variáveis também devem ser levadas em consideração, para Hölldobler e Wilson (1990), tanto a variação sazonal quanto a complexidade do ambiente são fatores predominantes nas mudanças diárias e mensais do horário de atividade de forrageamento das formigas.

O método utilizado, também interfere no resultado, no presente estudo, as armadilhas de solo tipo pitfall coletaram principalmente espécies com maior mobilidade no ambiente, sendo assim, em um ambiente mais frio, haveria a diminuição da riqueza observada de espécies de formigas (VARGAS et al., 2007). Este fato pode explicar a menor riqueza de

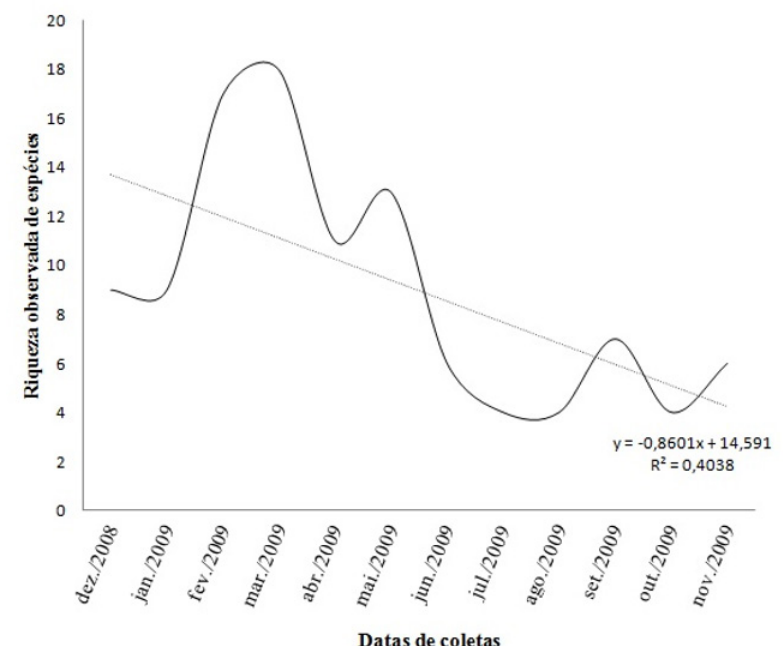

FIGURA 4: Riqueza observada de espécies de Formicidae (Hymenoptera) associadas a um povoamento, pós-incêndio, de Pinus elliottii, em Santa Maria - RS, Brasil (2008/2009).

FIGURE 4: Species richness observed of Formicidae (Hymenoptera) associated with a settlement, after fire, Pinus elliottii in Santa Maria, RS state, Brazil (2008/2009). 
espécies encontradas nos meses frios de inverno, período de junho a setembro (Figura 4).

Com base nas discussões propostas acerca dos resultados do levantamento e da análise comportamental das espécies encontradas neste trabalho, pode-se inferir que houve uma alteração na riqueza de espécies de formigas epigeicas após a ocorrência do fogo, principalmente, pela maior oferta imediata de alimentos para Pachycondyla striata e Acromyrmex crassispinus. Ressalta-se que, para avaliar os efeitos em longo prazo, é necessário revisitar o local e realizar novos estudos.

\section{CONCLUSÕES}

O fogo é um agente de distúrbio ambiental por vezes significativo, causando efeitos positivos e negativos sobre a fauna edáfica. Sua ação pode ter gerado efeito indireto negativo sobre Crematogaster victima e positivo sobre Pseudomyrmex termitarius, Acromyrmex crassispinus, Pachycondyla striata e Camponotus blandus presentes no sub-bosque de Pinus elliottii, pós-incêndio. Para um melhor entendimento sobre os possíveis impactos do fogo sobre essas espécies, sugerem-se estudos futuros em áreas acometidas por incêndios.

\section{AGRADECIMENTOS}

Ao Departamento de Defesa Fitossanitária da Universidade Federal de Santa Maria pelo apoio na realização do trabalho, disponibilizando espaço físico, materiais e produtos utilizados.

\section{REFERÊNCIAS BIBLIOGRÁFICAS}

ALMEIDA, L. M.; RIBEIRO-COSTA, C. S.; MARINONI, L. Manual de coleta, conservação, montagem e identificação de insetos. Ribeirão Preto: Holos, 2003. 88 p.

ARAÚJO, E. A.; RIBEIRO, G. A. Impactos do fogo sobre a entomofauna do solo em ecossistemas florestais. Natureza \& Desenvolvimento, Viçosa, v. 1, n. 1, p. 75-85, 2005.

BOLTON, B. Identification guide to the ant genera of the world. Cambridge: Harvard University Press, 1994. $222 \mathrm{p}$.

BRASIL. Lei № 9.605, de 12 de fevereiro de 1998. Dispõe sobre as sanções penais e administrativas derivadas de condutas e atividades lesivas ao meio ambiente, e dá outras providências. Diário Oficial [da] República Federativa do Brasil, Brasília, DF,
17 fev. 1998.

COELHO, I. R.; COUTO-SANTOS, F. R.; KRETTLI, L. G. Efeito do fogo sobre os padrões de co-ocorrência de formigas na Serra do Cipó, Brasil. In: CONGRESSO DE ECOLOGIA DO BRASIL, 8., 2007, Caxambu. Anais... Caxambu, 2007. p. 1-2. COLWELL, R. K. EstimateS: estatistical estimation of species richness and shared species from samples. Version 7.5.2. 2009. Disponível em: $<$ http://viceroy.eeb.uconn.edu/estimates $>$. Acesso em: 23 nov. 2011.

CORREIA, M. E. F.; PINHEIRO, L. B. A. Monitoramento da fauna de solo sob diferentes coberturas vegetais em um sistema integrado de produção agrícola, Seropédica $(\mathbf{R J})$. Seropédica: EMBRAPA Agrobiologia, 1999, 15 p. (EmbrapaCNPAB. Circular técnica, 3).

FRIZZO, T. L. M. et al. Revisão dos efeitos do fogo sobre a fauna de formações savânicas do Brasil. Oecologia Australis, Rio de Janeiro, v. 15, n. 2, p. 365-379, 2011.

FRIZZO, T. L. M.; CAMPOS, R. I.; VASCONCELOS, H. L. Efeito do fogo sobre a riqueza e abundância de formigas em área de Cerrado no Brasil Central. Biológico, São Paulo, v. 69, Suplemento 2, p. 275-278, 2007.

GIANOTTI, E.; MACHADO, V. L. L. Notes on the foraging of two species of ponerine ants: food resources and daily hunting activities (Hymenoptera, Formicidae). Bioikos, Campinas, v. 6, n. (1/2), p. 7-17, jan./dez. 1994. HÖLLDOBLER, B.; WILSON, E. O. The ants. Cambridge, Massachusetts: Harvard University Press, 1990. $732 \mathrm{p}$.

KLEINPAUL, I. S. et al. Suficiência amostral para coletas de serapilheira acumulada sobre o solo em Pinus elliottii Engelm, Eucalyptus sp. e floresta estacional decidual. Revista Árvore, Viçosa, v. 29, n.6, p. 965-972, nov./dez. 2005.

LATTKE, J. E. Subfamilia Ponerinae. In: FERNÁNDEZ, F. (Ed.). Introducción a las hormigas de la región Neotropical. Bogotá: Acta Nocturna, 2003. p. 261-276.

LINK, D.; LINK, F. M.; OLIVEIRA, A. A. de. Potencia de dano da formiga preta, Acromyrmex crassispinus, em mudas de eucalipto e de pinus. In: SIMPÓSIO LATINO-AMERICANO SOBRE MANEJO FLORESTAL, 2., 2001, Santa Maria. Anais... Santa Maia: UFSM, 2001. p. 671-676.

LONGINO, J. T. What to do with the data. In: AGOSTI, D.; MAJER, J. D.; ALONSO, L. E.; SCHULTZ, T. R. (Ed). Ants: Standard Methods 
for Measuring and Monitoring Biodiversity. Washington, London: Smithsonian Institution Press, 2000. p.186-203.

LOPES, C. T. et al. Efeitos do fogo e da sazonalidade sobre formigas em três espécies arbóreas do Cerrado do Brasil Central. Biológico, São Paulo, v. 69, Suplemento 2, p. Xx-Xx, 2007.

LORO, L. V.; HIRAMATSU, N. A. Comportamento do fogo, em condições de laboratório, em combustíveis provenientes de um povoamento de Pinus elliottii L. Floresta, Curitiba, v. 34, n. 2, p. 127-130, mai./ago. 2004.

MAJER, J. D. Ant recolonization of rehabilitated bauxite mines at Trombetas, Pará, Brazil. Journal of Tropical Ecology, Winchelsea, v. 12, p. 257-273, 1996.

MAJER, J. D. Ants: Bio-indicators of minesite rehabilitation, land-use, and land conservation. Environmental Management, New York, v. 7, n. 4, p. 375-383, 1983. MAJER, J. D. The use of pitfall traps for sampling ants - a critique. Memoirs of Museum Victoria, Melbourne, v. 56, n. 2, p. 323-329, 1997.

MARCHIORI, J. N. C. Dendrologia das angiospermas. Santa Maria: Ed. UFSM, 1996. $162 \mathrm{p}$.

MEDEIROS, F. N. da S. Ecologia comportamental da formiga Pachycondyla striata Fr. Smith (Formicidae: Ponerinae) em uma floresta do Sudeste do Brasil. 1997. 70 f. Dissertação (Mestrado em Ciências Biológicas) - Universidade Estadual de Campinas, Campinas, 1997.

MILLER, R. B; STOUT, J. B.; LEE, K. E. Biological and chemical changes following scrub burning on a New Zealand hill soil. New Zealand Journal of Science Technology, New Zealand, v. 37, p. 290-313, 1955.

MOREnO, J. A. Clima do Rio Grande do Sul. Porto Alegre: Secretaria da Agricultura, 1962. 42 p. NUNES, L. A. P. L.; ARAÚJO FILHO, J. A.; MENEZES, R. I. Q. Diversidade da fauna edáfica em solos submetidos a diferentes sistemas de manejo no semi-árido nordestino. Scientia Agraria, Curitiba, v. 10, n. 1, p. 43-49, 2009.

OLIVEIRA, E. P.; FRANKLIN, E. Efeito do fogo sobre a mesofauna do solo: Recomendações em áreas queimadas. Pesquisa Agropecuária Brasileira, Brasília, v. 28, n. 3, p. 357-369, mar. 1993.

PALMER, M. W. The estimation of species richness by extrapolation. Ecology, New York, v. 71, n. 3, p. 1195-1198, jun. 1990.
PEREIRA, P. R. B. et al. Contribuição à geografia física do município de Santa Maria: unidades de paisagem. Geografia, Ensino \& Pesquisa, Santa Maria, v. 3, p. 37-68, 1989.

PES, L. et al. Comportamento da erva-mate (Ilex paraguariensis St.Hil.) em consórcio silvicultural. Ciência Florestal, Santa Maria, v. 5, n. 1, p. 19-32, 1995.

PETERNELLI, E. F. de O.; DELLA LUCIA, T. M. C.; MARTINS, S. V. Espécies de formigas que interagem com as sementes de Mabea fistulifera Mart. (EUPHORBIACEAE). Revista Árvore, Viçosa, v. 28, n. 5, p. 733-738, set./out. 2004.

PIPPI, M. C.; KRIEGER, J. M.; HOPPE, J. M. Quantificação do material combustível na serapilheira e vegetação de sub-bosque e inventário florestal de uma floresta de Pinus elliottii aos 9 anos. In: CONGRESSO FLORESTAL ESTADUAL DE NOVA PRATA, 8., 2000. Nova Prata. Anais... Nova Prata, 2000.

RANDO, J. S. S.; FORTI, L. C. Ocorrência de formigas Acromyrmex Mayr, 1865, em alguns municípios do Brasil. Acta Scientiarum Biological Sciences, Maringá, v.27, n. 2, p.129-133, abr./jun. 2005.

RIBAS, C. R. et al. Ants as indicators in Brazil: A review with suggestions to improve the use of ants in environmental monitoring programs. Psyche, p. 1-23, 2012.

RICKLEFS, R. E. A Economia da Natureza. Rio de Janeiro: Guanabara Koogan, 1996. 470 p.

ROMERO, H.; JAFFÉ, K. A comparison of methods for sampling ants (Hymenoptera, Formicidae) in savannas. Biotropica, Kansas, v. 21, n. 4, p. 348-352, 1989.

SANTOS, G. M. de M.; RESENDE, J. J. Predação de Syntermes molestus (Burmeister, 1839) (IsopteraTermitidae) por Camponotus blandus (Fr. Smith, 1858) (Hymenoptera-Formicidae) em Feira de Santana-Ba. Sitientibus, Feira de Santana, n. 15, p. 175-182, 1996.

SANTOS, J. C.; DELABIE, J. H. C.; FERNANDES, G. W. A 15-year post evaluation of the fire effects on ant community in an area of Amazonian forest.

Revista Brasileira de Entomologia, Curitiba, v. 52, n. 1, p. 82-87, mar. 2008.

SARMIENTO-M, C. E. Metodologías de captura y estudio de las hormigas. In: FERNÁNDEZ, F. (Ed.). Introducción a las hormigas de la región Neotropical. Bogotá: Acta Nocturna, 2003. p. 201-210.

SHIMIZU, J. Y. Pinus na silvicultura brasileira. 
Revista da Madeira, Curitiba, v. 16, n. 99, p. 4-14, set. 2006.

SILVESTRE, R. Estrutura de comunidades de formigas do Cerrado. 2000. 216 f. Tese (Doutorado em Ciências) - Universidade de São Paulo, Ribeirão Preto, 2000.

SILVESTRE, R.; SILVA, R. R. Guildas de formigas da Estação Ecológica Jataí, Luis Antonio-SP sugestões para aplicação de guildas como bioindicadores ambientais. Biotemas, Florianópolis, v. 14, n. 1, p. 37-69, 2001.

VARGAS, A. B. et al. Efeitos de Fatores Ambientais sobre a Mirmecofauna em Comunidade de Restinga no Rio de Janeiro, RJ. Neotropical Entomology,
Londrina, v. 36, n. 1, p. 28-37, jan./fev. 2007.

VASCONCELOS, H. L. et al. Dynamics of the Leaf-Litter Arthropod fauna following fire in a Neotropical Woodland Savanna. PLoS ONE, United Kingdom, v. 4, n. 11, p.1-9, nov. 2009.

WILSON, E. O. Which are the most prevalent ant genera? Studia Entomologica, Petrópolis, v. 19, p.187-200, 1976.

YORK, A. The long-term effects of fire on Forest ant communities: managenent implications for the conservation of diversity. Memoirs of the Queensland Museum, Queensland, v. 36, p. 231-239, 1994. 